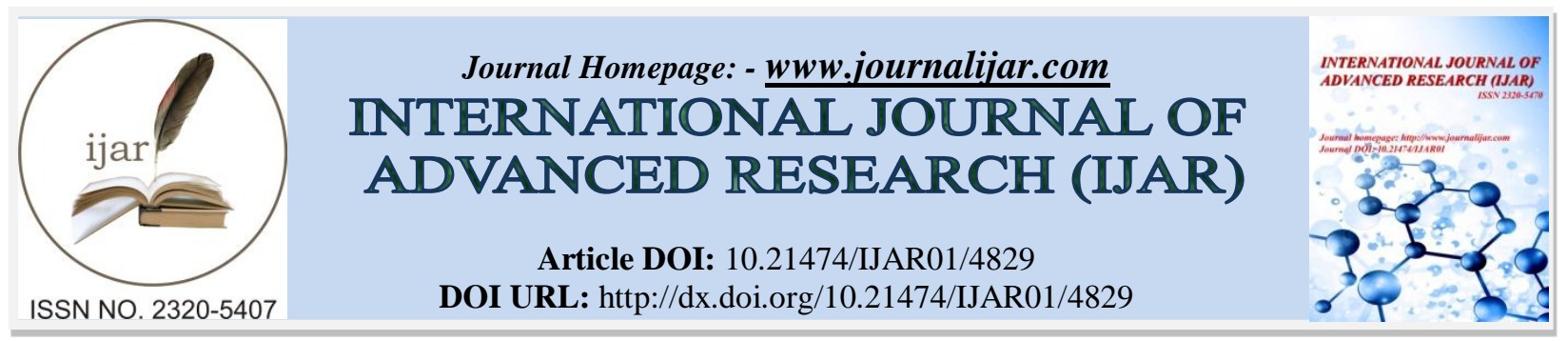

RESEARCH ARTICLE

\title{
CARDIAC CONDUCTION DEFECTS AND CARDIOVASCULAR RISK FACTORS -A HOSPITAL BASED STUDY.
}

Aadil Ashraf ${ }^{1}$, Touseef ahmad Mir ${ }^{1}$, Ahmad Wajeed Yousuf ${ }^{1}$, Gazzanfar Ali $^{2}$, Javed Khan ${ }^{1}$ and Irfan Gul $^{1}$.

1. Senior Resident, Deptt. Of Medicine, Govt. Medical College, Srinagar.

2. Professor, Deptt. Of Medicine, Govt. Medical College, Srinagar.

\section{Manuscript Info}

Manuscript History

Received: 12 May 2017

Final Accepted: 14 June 2017

Published: July 2017

Key words:-

Cardiac conduction defects ( LBBB, CHB, AV BLOCKS), hypertension, coronary artery disease, diabetes, risk factors for conduction defects.

\section{Abstract}

Background: The interest in cardiac conduction system has focused primarily on its role as a predictor of mortality and coexistent cardiovascular diseases particularly hospitalised patients.

Objectives: The study was undertaken to study the association of cardiac conduction defects with underlying cardiovascular diseases and various risk factors.

Methodology: The study was conducted from 1st March 2012 to 31st august 2013 and included cases $>20 y r s$ of age presenting to OPD or admitted in SMHS hospital and showing some form of cardiac conduction defect on a standard 12 lead ECG. A total of 1710 cases were studied. A thorough medical history and meticulous physical examination was done and relevant investigations ordered to find out the presence of underlying cardiovascular diseases and risk factors

Results: Of the 1710 cases, 990(57.9\%) were males and 720(42.1\%) were females. Most of cases were seen in the age group of 70-79yrs (25.7\%). Risk factors including Hypertension was found in 1095(64\%) cases, Coronary Artery Disease(CAD) in 445(26\%), dyslipidemia in $429(25 \%)$, diabetes in $410(23.9 \%)$ and gout in $51(2.9 \%)$ cases. $1270(74 \%)$ cases were smokers, 632(36.9\%) were having sedentary life style and 496(29\%) cases were obese/overweight.

Conclusion: Hypertension, smoking, CAD, obesity and sedentary life style are the major risk factors.

Copy Right, IJAR, 2017,. All rights reserved.

\section{Introduction:-}

The ECG has evolved into an extremely useful clinical laboratory tool and is the only practical means of recording the electrical behaviour of heart. Its usefulness as a diagnostic method is the result of careful, often purely deductive analysis of innumerable patient records and of studies correlating the ECG with basic electrophysiological properties of heart; with clinical and laboratory findings, and with anatomical, pathological and experimental observations. The result has been that electrocardiography can be used, within limits, to identity anatomical, metabolic, ionic and hemodynamic changes. It is often an independent marker of cardiac disease and occasionally the only indicator of a Pathological process. ${ }^{1}$ 
Cardiac muscle has the property of automatic impulse formation and rhythmic contraction; impulses are generated in specialized tissue that forms the atrioventricular (AV) conduction system. The conduction system consists of Sinoatrial-node (SA node) (which itself consists of $\mathrm{P}$ cells that form the impulse), transitional cells that transmit the impulse through the node, and collagen fibres, interatrial conduction "pathways" (or tracts), the A.V node, the bundle of His, the right and left bundle branches, the fascicles of the left bundle branch (Anterosuperior, inferoposterior, and septal) and the distal Purkinje system. ${ }^{2}$

The conduction system can be involved because of various cardiac diseases which can interfere with the formation and the conduction of electrical impulses, like coronary heart diseases, hypertension, diabetes mellitus, obesity, aortic value disease and various other diseases which affect this system primarily or secondarily like cardiomyopathies, amyloidosis, myocarditis, scleroderma, muscular dystrophies, metabolic abnormalities and some congenital diseases like endocardial cushion defects etc. ${ }^{3}$ This system can be involved by degenerative processes which is known as fibrocalcific degenerative disease (Lengres disease) of conduction system. ${ }^{4}$

The interest in Bundle Branch Blocks has focused primarily on its role as a predictor of mortality and coexistent cardiovascular diseases.

This type of study has not been done in the state so far. Hence this study was undertaken in this Hospital to establish the presence or absence of different cardiovascular risk factors with these cardiac conduction defects.

Aims:-

To Study the relationship of Cardiac Conduction Defects with underlying cardiovascular disease and risk factors.

\section{Material And Methods:-}

This study included all the patients admitted in medical and surgical wards of SMHS hospital with some form of ECG documented Cardiac conduction abnormality irrespective of their underlying illness.

This study was conducted from a period of $1^{\text {st }}$ of March 2012 to 31st of August 2013. A detailed History was taken from the patients regarding the presenting illness, underlying illness and drug history.

A thorough general physical examination was done followed by a meticulous systemic examination with particular stress on Cardio respiratory system. Subjects were weighed without shoes on a weighing machine in kilograms and Height was measured in meters without shoes by making the subjects to stand against a wall on a firm level ground, wall being already marked with a measuring tape.BMI was calculated as per the following formula (Quetlets formulae) $)^{5}$ :

$\mathrm{BMI}=$ Weight in $\mathrm{kgs} /(\text { Height })^{2}$ in meters.

Blood pressure was recorded in subjects in the right upper arm with a mercury sphygmomanometer in mmHg after making the patient to relax for five minutes. Complete Blood Count, Blood sugar, KFT/Na/K, Routine Urine examination, Chest XRAY, Lipid Profile, Liver function test, lipid profile and Serum Uric acid were routinely done in all patients.

A standard 12 lead ECG was taken with a paper speed of $25 \mathrm{~mm} / \mathrm{sec}$. In addition a one minute rhythm strip was also recorded.

The different cardiac conduction defects were diagnosed using the definitive criteria. ${ }^{3,6,7,8}$

All the patients were screened for cardiovascular risk factors as per defined criteria:

1. Hypertension: was considered to be present if the patient was taking anti-hypertensive medication at the time of presentation or If blood pressure recorded was equal to or greater than $140 \mathrm{~mm} \mathrm{Hg}$ systolic and, equal to or greater than $90 \mathrm{~mm} \mathrm{Hg}$ Diastolic, at least on two separate occasions.

2. Diabetes_Mellitus: was defined as patients diagnosed on the basis of fasting glucose $126 \mathrm{mg} / \mathrm{dI}$ or Serum HbA1c $>6.5 \%$ or symptoms of diabetes plus random blood glucose $200 \mathrm{mg} / \mathrm{d} 1$ or patients on anti-diabetic drugs..

3. Coronary_Artery_Disease(Cad) : was defined as patients with a history of stable angina, unstable angina, Myocardial infarction(New/old), angiographic evidence of Coronary Artery disease. 
4. Smoking: was defined as per CDC guidelines as any patient who had smoked at least 100 cigarettes in his/her entire life time and who at the time of survey smoked either everyday or some days.

5. Sedentary_Life_Style: was defined after using the standard International Physical Activity Questionnaire(IPAQ), Revised November 2005. ${ }^{9}$

6. Obesity: was defined as BMI of $\geq 25 \mathrm{~kg} / \mathrm{m}^{2}$ as per revised criteria for Asian Indians. ${ }^{10,11}$

7. Dyslipidemia : was defined was if total Cholesterol was equal to or greater than $200 \mathrm{mg} / \mathrm{dl}$,LDL cholesterol equal to or greater than $130 \mathrm{mg} / \mathrm{dl}$, HDL cholesterol less than $40 \mathrm{mg} / \mathrm{dl}$ and Serum Triglycerides $150 \mathrm{mg} / \mathrm{dl}$ or combination of these criteria ${ }^{5}$.

8. Hyperuricemia: was defined as patients with an acute gout, serum uric acid more than $6.8 \mathrm{mg} / \mathrm{d} 1$ in men and $6 \mathrm{mg} / \mathrm{dI}$ in women or already on treatment tor hyperuricemia. ${ }^{5}$

Statistical_Methods: Statistical testing was conducted with the statistical package for the social science system version SPSS 20.0.Continuous variables are presented as mean \pm SD and categorized into groups; Categorical variables are presented as frequencies and percentage. Nominal categorical data between the groups were compared using Chi-square test or Fisher's exact test as appropriate. $p<0.05$ was considered statistically significant.

\section{Results:-}

Table 1:- Showing Number and Percentage of Cases in Relation to Sex.

\begin{tabular}{|l|l|l|}
\hline SEX & NUMBER (n) & PERCENT (\%) \\
\hline MALE & 990 & 57.9 \\
\hline FEMALE & 720 & 42.1 \\
\hline Total & 1710 & 100.0 \\
\hline
\end{tabular}

A total of 1710 cases were studied. Out of which 990 (57.9\%) were males and 720(42.1\%) were females.

Table 2:- Showing distribution of studied cases in relation to age and sex.

\begin{tabular}{|c|c|c|c|c|c|}
\hline \multirow{3}{*}{ AGE (years) } & \multicolumn{4}{|c|}{ SEX } & \multirow{3}{*}{ Total } \\
\hline & \multicolumn{2}{|c|}{ MALE } & \multicolumn{2}{|c|}{ FEMALE } & \\
\hline & $\mathrm{n}$ & $\%$ & $\mathrm{n}$ & $\%$ & \\
\hline $20-29$ & 35 & $70.0 \%$ & 15 & $30.0 \%$ & 50 \\
\hline $30-39$ & 75 & $57.7 \%$ & 55 & $42.3 \%$ & 130 \\
\hline $40-49$ & 146 & $63.5 \%$ & 84 & $36.5 \%$ & 230 \\
\hline $50-59$ & 140 & $45.5 \%$ & 168 & $54.5 \%$ & 308 \\
\hline $60-69$ & 233 & $60.4 \%$ & 153 & $39.6 \%$ & 386 \\
\hline $70-79$ & 279 & $60.9 \%$ & 179 & $39.1 \%$ & 458 \\
\hline $80-89$ & 70 & $54.7 \%$ & 58 & $45.3 \%$ & 128 \\
\hline$>90$ & 12 & $60.0 \%$ & 8 & $40.0 \%$ & 20 \\
\hline Total & 990 & $57.9 \%$ & 720 & $42.1 \%$ & 1710 \\
\hline
\end{tabular}

Among males, most of the studied cases were in 70-79 years age group: 279(28.18\%) and among females most of the studied cases were in the age group of 70-79 years: $179(24.86 \%)$. Mean age among males was (62 \pm 16$)$ years with minimum and maximum age of (22 and 94) years respectively. Mean age among females was (62 \pm 15$)$ years with minimum and maximum age of 23 and 95 years respectively. There was no statistical significance between age of males and females $(\mathrm{p}=0.88$ ). Males were more as compared to females in all the age groups except 50-59 year age group ( $54.5 \%$ females vs $45.5 \%$ males).

Table 3:- Showing the number and percentage of different cardiac conduction defects in relation to sex.

\begin{tabular}{|l|l|l|l|l|l|l|}
\hline \multirow{2}{*}{ DIAGNOSIS } & \multicolumn{5}{|l|}{ SEX } \\
\cline { 2 - 7 } & \multicolumn{2}{|l|}{ MALE } & \multicolumn{4}{l|}{ FEMALE } \\
\cline { 2 - 7 } & $\mathbf{n}$ & Row \% & Column \% & $\mathbf{n}$ & Row \% & Column \% \\
\hline $1^{\text {st }}$ DEGREE & 23 & $67.6 \%$ & $2.3 \%$ & 11 & $32.4 \%$ & $1.5 \%$ \\
\hline $2^{\text {nd }}$ DEGREE TYPE 1 & 42 & $64.6 \%$ & $4.2 \%$ & 23 & $35.4 \%$ & $3.2 \%$ \\
\hline $2^{\text {nd }}$ DEGREE TYPE 2 & 13 & $50.0 \%$ & $1.3 \%$ & 13 & $50.0 \%$ & $1.8 \%$ \\
\hline CHB & 65 & $62.5 \%$ & $6.6 \%$ & 39 & $37.5 \%$ & $5.4 \%$ \\
\hline LAHB & 257 & $59.6 \%$ & $26.0 \%$ & 174 & $40.4 \%$ & $24.2 \%$ \\
\hline LBBB & 274 & $54.7 \%$ & $27.7 \%$ & 227 & $45.3 \%$ & $31.5 \%$ \\
\hline
\end{tabular}




\begin{tabular}{|l|l|l|l|l|l|l|}
\hline LPHB & 3 & $42.9 \%$ & $0.3 \%$ & 4 & $57.1 \%$ & $0.6 \%$ \\
\hline RBBB & 171 & $55.5 \%$ & $17.3 \%$ & 137 & $44.5 \%$ & $19.0 \%$ \\
\hline RBBB + LAH & 99 & $61.5 \%$ & $10.0 \%$ & 62 & $38.5 \%$ & $8.6 \%$ \\
\hline RBBB + LPH & 6 & $50.0 \%$ & $0.6 \%$ & 6 & $50.0 \%$ & $0.8 \%$ \\
\hline SI SII SIII & 7 & $53.8 \%$ & $0.7 \%$ & 6 & $46.2 \%$ & $0.8 \%$ \\
\hline TFB & 30 & $62.5 \%$ & $3.0 \%$ & 18 & $37.5 \%$ & $2.5 \%$ \\
\hline Total & 990 & $57.9 \%$ & $100.0 \%$ & 720 & $42.1 \%$ & $100.0 \%$ \\
\hline
\end{tabular}

LBBB was the commonest conduction defect in both the sexes; $27.7 \%$ in males and $31.5 \%$ in females. In all the conduction defects, males were more as compared to females except in LPHB where females outnumbered males (57\% vs 43\%). There was no statistical significance between males and females with regard to the conduction abnormalities $(\mathrm{p}=0.58)$.

Table 4:- Showing the distribution of the studied cases as per the presence or absence of hypertension.

\begin{tabular}{|c|c|c|c|c|c|c|c|c|c|c|}
\hline \multirow{2}{*}{$\begin{array}{l}\text { Hypertensio } \\
\text { n }\end{array}$} & \multicolumn{3}{|c|}{ Male } & \multicolumn{3}{|c|}{ Female } & \multicolumn{3}{|c|}{ Total } & \\
\hline & $\mathbf{n}$ & $\begin{array}{l}\text { Row } \\
\%\end{array}$ & $\begin{array}{l}\text { Colum } \\
\text { n \% }\end{array}$ & $\mathbf{n}$ & $\begin{array}{l}\text { Row } \\
\%\end{array}$ & $\begin{array}{l}\text { Colum } \\
\text { n \% }\end{array}$ & $\mathrm{n}$ & $\begin{array}{l}\text { Row } \\
\%\end{array}$ & $\begin{array}{l}\text { Colum } \\
\text { n \% }\end{array}$ & \multirow{4}{*}{$\begin{array}{l}0.000 \\
1\end{array}$} \\
\hline Yes & $\begin{array}{l}67 \\
3 \\
\end{array}$ & $\begin{array}{l}61.5 \\
\%\end{array}$ & $68.0 \%$ & $\begin{array}{l}42 \\
2\end{array}$ & $\begin{array}{l}38.5 \\
\%\end{array}$ & $58.6 \%$ & $\begin{array}{l}109 \\
5\end{array}$ & $\begin{array}{l}100.0 \\
\%\end{array}$ & $64.0 \%$ & \\
\hline No & $\begin{array}{l}31 \\
7\end{array}$ & $\begin{array}{l}51.5 \\
\%\end{array}$ & $32.0 \%$ & $\begin{array}{l}29 \\
8\end{array}$ & $\begin{array}{l}48.5 \\
\%\end{array}$ & $41.4 \%$ & 615 & $\begin{array}{l}100.0 \\
\%\end{array}$ & $36.0 \%$ & \\
\hline Total & $\begin{array}{l}99 \\
0\end{array}$ & $\begin{array}{l}57.9 \\
\%\end{array}$ & $100.0 \%$ & $\begin{array}{l}72 \\
0\end{array}$ & $\begin{array}{l}42.1 \\
\%\end{array}$ & $100.0 \%$ & $\begin{array}{l}171 \\
0\end{array}$ & $\begin{array}{l}100.0 \\
\%\end{array}$ & $100.0 \%$ & \\
\hline
\end{tabular}

Of the studied cases, 1095 (64\%) were hypertensive. Males were more $673(68 \%)$ cases as compared to females $422(58.6 \%)$ and the difference was statistically significant $(\mathrm{p}=0.04)$. The presence of hypertension in the studied population was found to be highly significant $(\mathrm{p}<0.0001)$.

Table 5:- Showing the distribution and percentage of hypertension in different cardiac conduction defects in studied cases.

\begin{tabular}{|c|c|c|c|c|}
\hline \multicolumn{2}{|c|}{ DIAGNOSIS } & \multicolumn{2}{|c|}{ HYPERTENSION } & \multirow{3}{*}{$\begin{array}{c}\text { Total } \\
34\end{array}$} \\
\hline & & YES & NO & \\
\hline \multirow[t]{2}{*}{ 1st DEGREE } & $\mathrm{n}$ & 1 & 33 & \\
\hline & Row \% & $2.9 \%$ & $97.1 \%$ & $100.0 \%$ \\
\hline \multirow[t]{2}{*}{ 2nd DEGREE TYPE 1} & $\mathrm{n}$ & 22 & 43 & 65 \\
\hline & Row \% & $33.8 \%$ & $66.2 \%$ & $100.0 \%$ \\
\hline \multirow[t]{2}{*}{ 2nd DEGREE TYPE 2} & $\mathrm{n}$ & 19 & 7 & 26 \\
\hline & Row \% & $73.1 \%$ & $26.9 \%$ & $100.0 \%$ \\
\hline \multirow[t]{2}{*}{ CHB } & $\mathrm{n}$ & 98 & 6 & 104 \\
\hline & Row \% & $94.2 \%$ & $5.8 \%$ & $100.0 \%$ \\
\hline \multirow[t]{2}{*}{ LAHB } & $\mathrm{n}$ & 276 & 155 & 431 \\
\hline & Row \% & $64.0 \%$ & $36.0 \%$ & $100.0 \%$ \\
\hline \multirow[t]{2}{*}{ LBBB } & $\mathrm{n}$ & 422 & 79 & 501 \\
\hline & Row \% & $84.2 \%$ & $15.8 \%$ & $100.0 \%$ \\
\hline \multirow[t]{2}{*}{ LPHB } & $\mathrm{n}$ & 5 & 2 & 7 \\
\hline & Row $\%$ & $71.4 \%$ & $28.6 \%$ & $100.0 \%$ \\
\hline \multirow[t]{2}{*}{ RBBB } & $\mathrm{n}$ & 75 & 233 & 308 \\
\hline & Row $\%$ & $24.4 \%$ & $75.6 \%$ & $100.0 \%$ \\
\hline \multirow[t]{2}{*}{ RBBB + LAH } & $\mathrm{n}$ & 122 & 39 & 161 \\
\hline & Row $\%$ & $75.8 \%$ & $24.2 \%$ & $100.0 \%$ \\
\hline \multirow[t]{2}{*}{$\mathrm{RBBB}+\mathrm{LPH}$} & $\mathrm{n}$ & 7 & 5 & 12 \\
\hline & Row $\%$ & $58.3 \%$ & $41.7 \%$ & $100.0 \%$ \\
\hline \multirow[t]{2}{*}{ SI SII SIII } & $\mathrm{n}$ & 4 & 9 & 13 \\
\hline & Row \% & $30.8 \%$ & $69.2 \%$ & $100.0 \%$ \\
\hline
\end{tabular}




\begin{tabular}{|c|c|c|c|c|}
\hline \multirow{2}{*}{ TFB } & $\mathrm{n}$ & 44 & 4 & 48 \\
\cline { 2 - 5 } & Row $\%$ & $91.7 \%$ & $8.3 \%$ & $100.0 \%$ \\
\hline Total & $\mathrm{n}$ & 1095 & 615 & 1710 \\
\hline
\end{tabular}

The percentage of hypertension in different cardiac conduction defects in decreasing order is as under:

I. Complete heart block (CHB):

II. Trifascicular heart block(TFB):

III. Left bundle branch block( LBBB):

IV. Bifascicular block ( RBBB $+\mathrm{LAH}, \mathrm{RBBB}+\mathrm{LPH})$ :

V. $\quad 2^{\text {nd }}$ degree type 2 :

VI. Left posterior hemiblock( LPHB):

VII. Left anterior hemiblock ( LAHB):

VIII. $\quad 2^{\text {nd }}$ degree type 1 :

IX. SI SII SIII :

X. Right bundle branch block( RBBB):

XI. $1^{\text {st }}$ degree heart block :
$94.2 \%$

$91.7 \%$

$84.2 \%$

$74.5 \%$

$73.1 \%$

$71.4 \%$

$64 \%$

$33.8 \%$

$30.8 \%$

$24.4 \%$

$2.9 \%$

Table 6:- Showing the distribution of the studied cases as per the presence or absence of diabetes mellitus.

\begin{tabular}{|c|c|c|c|c|c|c|c|c|c|c|}
\hline \multirow[t]{2}{*}{ Diabetes } & \multicolumn{3}{|c|}{ Male } & \multicolumn{3}{|c|}{ Female } & \multicolumn{3}{|c|}{ Total } & \\
\hline & $\mathbf{n}$ & $\begin{array}{l}\text { Row } \\
\%\end{array}$ & $\begin{array}{l}\text { Column } \\
\% \\
\end{array}$ & $\mathbf{n}$ & $\begin{array}{l}\text { Row } \\
\%\end{array}$ & $\begin{array}{l}\text { Column } \\
\%\end{array}$ & $\mathbf{n}$ & $\begin{array}{l}\text { Row } \\
\%\end{array}$ & $\begin{array}{l}\text { Column } \\
\%\end{array}$ & \multirow{4}{*}{0.029} \\
\hline YES & 218 & $53.2 \%$ & $22.0 \%$ & 192 & $46.8 \%$ & $26.7 \%$ & 410 & $100.0 \%$ & $24.0 \%$ & \\
\hline NO & 772 & $59.4 \%$ & $78.0 \%$ & 528 & $40.6 \%$ & $73.3 \%$ & 1300 & $100.0 \%$ & $76.0 \%$ & \\
\hline Total & 990 & $57.9 \%$ & $100.0 \%$ & 720 & $42.1 \%$ & $100.0 \%$ & 1710 & $100.0 \%$ & $100.0 \%$ & \\
\hline
\end{tabular}

Of the studied cases, $410(24 \%)$ were diabetics. Males included 218 cases $(22 \%)$ and females included 192 cases $(26.7 \%)$. Females were more as compared to males with as statistical significance $(p=0.030)$. The association of diabetes mellitus with cardiac conduction defects was found to be significant $(\mathrm{p}=0.029)$.

Table 7:- Showing the distribution and percentage of diabetes mellitus in different cardiac conduction defects in studied cases.

\begin{tabular}{|c|c|c|c|c|}
\hline \multirow[t]{2}{*}{ Diagnosis } & \multicolumn{3}{|c|}{ Diabetes } & \multirow[t]{2}{*}{ Total } \\
\hline & \multicolumn{2}{|c|}{ Yes } & No & \\
\hline \multirow[t]{2}{*}{ 1st DEGREE } & $\mathrm{n}$ & 0 & 34 & 34 \\
\hline & Row \% & $0.0 \%$ & $100.0 \%$ & $100.0 \%$ \\
\hline \multirow[t]{2}{*}{ 2nd DEGREE TYPE 1} & $\mathrm{n}$ & 4 & 61 & 65 \\
\hline & Row \% & $6.2 \%$ & $93.8 \%$ & $100.0 \%$ \\
\hline \multirow[t]{2}{*}{ 2nd DEGREE TYPE 2} & $\mathrm{n}$ & 12 & 14 & 26 \\
\hline & Row \% & $46.2 \%$ & $53.8 \%$ & $100.0 \%$ \\
\hline \multirow[t]{2}{*}{ CHB } & $\mathrm{n}$ & 53 & 51 & 104 \\
\hline & Row \% & $51.0 \%$ & $49.0 \%$ & $100.0 \%$ \\
\hline \multirow[t]{2}{*}{ LAHB } & $\mathrm{n}$ & 56 & 375 & 431 \\
\hline & Row \% & $13.0 \%$ & $87.0 \%$ & $100.0 \%$ \\
\hline \multirow[t]{2}{*}{ LBBB } & $\mathrm{n}$ & 168 & 333 & 501 \\
\hline & Row $\%$ & $33.5 \%$ & $66.5 \%$ & $100.0 \%$ \\
\hline \multirow[t]{2}{*}{ LPHB } & $\mathrm{n}$ & 3 & 4 & 7 \\
\hline & Row \% & $42.9 \%$ & $57.1 \%$ & $100.0 \%$ \\
\hline \multirow[t]{2}{*}{ RBBB } & $\mathrm{n}$ & 27 & 281 & 308 \\
\hline & Row \% & $8.8 \%$ & $91.2 \%$ & $100.0 \%$ \\
\hline \multirow[t]{2}{*}{ RBBB + LAH } & $\mathrm{n}$ & 53 & 108 & 161 \\
\hline & Row \% & $32.9 \%$ & $67.1 \%$ & $100.0 \%$ \\
\hline \multirow[t]{2}{*}{ RBBB + LPH } & $\mathrm{n}$ & 6 & 6 & 12 \\
\hline & Row \% & $50.0 \%$ & $50.0 \%$ & $100.0 \%$ \\
\hline SI SII SIII & $\mathrm{n}$ & 2 & 11 & 13 \\
\hline
\end{tabular}




\begin{tabular}{|c|c|c|c|c|}
\hline & Row \% & $15.4 \%$ & $84.6 \%$ & $100.0 \%$ \\
\hline \multirow{2}{*}{ TFB } & $\mathrm{n}$ & 26 & 22 & 48 \\
\cline { 2 - 5 } & Row \% & $54.2 \%$ & $45.8 \%$ & $100.0 \%$ \\
\hline \multirow{2}{*}{ Total } & $\mathrm{n}$ & 410 & 1300 & 1710 \\
\cline { 2 - 5 } & Row \% & $24.0 \%$ & $76.0 \%$ & $100.0 \%$ \\
\hline
\end{tabular}

The percentage of diabetes mellitus in different cardiac conduction defects in decreasing order was as under:

I. Trifascicular heart block(TFB):

II. Complete heart block (CHB):

III. $\quad 2^{\text {nd }}$ degree type 2 :

IV. Left posterior hemiblock( LPHB):

V. $\quad$ Bifascicular block $(\mathrm{RBBB}+\mathrm{LAH}, \mathrm{RBBB}+\mathrm{LPH})$ :

VI. Left bundle branch block( LBBB):

VII. SI SII SIII :

VIII. Left anterior hemiblock ( LAHB):

IX. Right bundle branch block( RBBB):

X. $\quad 2^{\text {nd }}$ degree type 1 :

XI. $1^{\text {st }}$ degree heart block :

\section{$54.2 \%$}

$51.0 \%$

$46.2 \%$

$42.9 \%$

$34.1 \%$

$33.5 \%$

$15.4 \%$

$13.0 \%$

$8.8 \%$

$6.2 \%$

0

Table 8:- Showing the distribution of the studied cases as per the presence or absence of Coronary Artery Disease (CAD).

\begin{tabular}{|c|c|c|c|c|c|c|c|c|c|c|}
\hline \multirow[t]{3}{*}{ CAD } & \multicolumn{6}{|c|}{ SEX } & & & & \multirow{2}{*}{$P$ value } \\
\hline & \multicolumn{3}{|c|}{ MALE } & \multicolumn{3}{|c|}{ FEMALE } & \multicolumn{3}{|c|}{ Total } & \\
\hline & $\mathrm{n}$ & $\begin{array}{l}\text { Row } \\
\%\end{array}$ & $\begin{array}{l}\text { Column } \\
\%\end{array}$ & $\mathrm{n}$ & $\begin{array}{l}\text { Row } \\
\%\end{array}$ & $\begin{array}{l}\text { Column } \\
\%\end{array}$ & $\mathrm{n}$ & $\begin{array}{l}\text { Row } \\
\%\end{array}$ & $\begin{array}{l}\text { Column } \\
\%\end{array}$ & \multirow{4}{*}{0.03} \\
\hline Yes & 274 & $61.6 \%$ & $27.7 \%$ & 171 & $38.4 \%$ & $23.8 \%$ & 445 & $100.0 \%$ & $26.0 \%$ & \\
\hline No & 716 & $56.6 \%$ & $72.3 \%$ & 549 & $43.4 \%$ & $76.2 \%$ & 1265 & $100.0 \%$ & $74.0 \%$ & \\
\hline Total & 990 & $57.9 \%$ & $100.0 \%$ & 720 & $42.1 \%$ & $100.0 \%$ & 1710 & $100.0 \%$ & $100.0 \%$ & \\
\hline
\end{tabular}

Of the studied cases, $445(26 \%)$ were having Coronary Artery Disease. Males included $274(27.7 \%)$ cases and females included $171(23.8 \%)$ cases with no statistically significant difference between them $(\mathrm{p}=0.074)$. The presence of Coronary Artery Disease in the studied population was found to $b$ significant $(\mathrm{p}=0.03$ ).

Table 9:- Showing the distribution and percentage of Coronary Artery Disease (CAD) in different cardiac conduction defects in studied cases.

\begin{tabular}{|c|c|c|c|c|}
\hline \multicolumn{2}{|l|}{ DIAGNOSIS } & \multicolumn{2}{|l|}{ CAD } & \multirow{3}{*}{\begin{tabular}{|l} 
Total \\
34
\end{tabular}} \\
\hline & & YES & NO & \\
\hline \multirow[t]{2}{*}{$1^{\text {st }}$ DEGREE } & $\mathrm{n}$ & 0 & 34 & \\
\hline & Row $\%$ & $0.0 \%$ & $100.0 \%$ & $100.0 \%$ \\
\hline \multirow[t]{2}{*}{$2^{\text {nd }}$ DEGREE TYPE 1} & $\mathrm{n}$ & 5 & 60 & 65 \\
\hline & Row \% & $7.7 \%$ & $92.3 \%$ & $100.0 \%$ \\
\hline \multirow[t]{2}{*}{$2^{\text {nd }}$ DEGREE TYPE 2} & $\mathrm{n}$ & 8 & 18 & 26 \\
\hline & Row $\%$ & $30.8 \%$ & $69.2 \%$ & $100.0 \%$ \\
\hline \multirow[t]{2}{*}{ CHB } & $\mathrm{n}$ & 25 & 79 & 104 \\
\hline & Row \% & $24.0 \%$ & $76.0 \%$ & $100.0 \%$ \\
\hline \multirow[t]{2}{*}{ LAHB } & $\mathrm{n}$ & 105 & 326 & 431 \\
\hline & Row $\%$ & $24.4 \%$ & $75.6 \%$ & $100.0 \%$ \\
\hline \multirow[t]{2}{*}{ LBBB } & $\mathrm{n}$ & 174 & 327 & 501 \\
\hline & Row $\%$ & $34.7 \%$ & $65.3 \%$ & $100.0 \%$ \\
\hline \multirow[t]{2}{*}{ LPHB } & $\mathrm{n}$ & 4 & 3 & 7 \\
\hline & Row \% & $57.1 \%$ & $42.9 \%$ & $100.0 \%$ \\
\hline \multirow[t]{2}{*}{ RBBB } & $\mathrm{n}$ & 73 & 235 & 308 \\
\hline & Row $\%$ & $23.7 \%$ & $76.3 \%$ & $100.0 \%$ \\
\hline \multirow[t]{2}{*}{ RBBB + LAH } & $\mathrm{n}$ & 32 & 129 & 161 \\
\hline & Row \% & $19.9 \%$ & $80.1 \%$ & $100.0 \%$ \\
\hline
\end{tabular}




\begin{tabular}{|l|l|l|l|l|}
\hline \multirow{2}{*}{ RBBB + LPH } & $\mathrm{n}$ & 6 & 6 & 12 \\
\cline { 2 - 5 } & Row $\%$ & $50.0 \%$ & $50.0 \%$ & $100.0 \%$ \\
\hline \multirow{2}{*}{ SI SII SIII } & $\mathrm{n}$ & 2 & 11 & 13 \\
\cline { 2 - 5 } & Row \% & $15.4 \%$ & $84.6 \%$ & $100.0 \%$ \\
\hline \multirow{2}{*}{ TFB } & $\mathrm{n}$ & 11 & 37 & 48 \\
\cline { 2 - 5 } & Row \% & $22.9 \%$ & $77.1 \%$ & $100.0 \%$ \\
\hline \multirow{2}{*}{ Total } & $\mathrm{n}$ & 445 & 1265 & 1710 \\
\cline { 2 - 5 } & Row \% & $26.0 \%$ & $74.0 \%$ & $100.0 \%$ \\
\hline
\end{tabular}

The percentage of Coronary Artery Disease (CAD) in different cardiac conduction defects in decreasing order is as under:

I. Left posterior hemiblock( LPHB) :

$57.1 \%$

II. Left bundle branch block( LBBB) :

$34.7 \%$

III. $\quad 2^{\text {nd }}$ degree type 2 :

$30.8 \%$

IV. Left anterior hemiblock ( LAHB) :

$24.4 \%$

V. Complete heart block (CHB) :

$24.0 \%$

VI. Right bundle branch block( RBBB) : $23.7 \%$

VII. Trifascicular heart block(TFB) : $22.9 \%$

VIII. Bifascicular block ( RBBB +LAH, RBBB + LPH) : $21.9 \%$

IX. SI SII SIII : $\quad 15.4 \%$

X. $\quad 2^{\text {nd }}$ degree type 1: $\quad 7.7 \%$

XI. $\quad 1^{\text {st }}$ degree heart block: $\quad 0 \%$

Table 10:- Showing the distribution of the studied cases as per the presence or absence of smoking.

\begin{tabular}{|c|c|c|c|c|c|c|c|c|c|c|}
\hline \multirow{3}{*}{$\begin{array}{l}\text { SMOKIN } \\
\text { G }\end{array}$} & \multicolumn{9}{|c|}{ SEX } & \multirow{3}{*}{$\begin{array}{l}P \\
\text { val } \\
\text { ue }\end{array}$} \\
\hline & \multicolumn{3}{|c|}{ MALE } & \multicolumn{3}{|c|}{ FEMALE } & \multicolumn{3}{|c|}{ Total } & \\
\hline & $\mathbf{n}$ & $\begin{array}{l}\text { Row } \\
\%\end{array}$ & $\begin{array}{l}\text { Colum } \\
\text { n \% }\end{array}$ & $\mathrm{n}$ & $\begin{array}{l}\text { Row } \\
\%\end{array}$ & $\begin{array}{l}\text { Colum } \\
\text { n \% }\end{array}$ & $\mathbf{n}$ & Row \% & $\begin{array}{l}\text { Colum } \\
\text { n \% }\end{array}$ & \\
\hline YES & $\begin{array}{l}81 \\
1\end{array}$ & $\begin{array}{l}63.9 \\
\%\end{array}$ & $81.9 \%$ & $\begin{array}{l}45 \\
9\end{array}$ & $\begin{array}{l}36.1 \\
\%\end{array}$ & $63.8 \%$ & $\begin{array}{l}127 \\
0\end{array}$ & $100.0 \%$ & $74.3 \%$ & \multirow{3}{*}{$\begin{array}{l}0.0 \\
001\end{array}$} \\
\hline NO & $\begin{array}{l}17 \\
9\end{array}$ & $\begin{array}{l}40.7 \\
\%\end{array}$ & $18.1 \%$ & $\begin{array}{l}26 \\
1\end{array}$ & $\begin{array}{l}59.3 \\
\%\end{array}$ & $36.2 \%$ & 440 & $100.0 \%$ & $25.7 \%$ & \\
\hline Total & $\begin{array}{l}99 \\
0\end{array}$ & $\begin{array}{l}57.9 \\
\%\end{array}$ & $100.0 \%$ & $\begin{array}{l}72 \\
0\end{array}$ & $\begin{array}{l}42.1 \\
\%\end{array}$ & $100.0 \%$ & $\begin{array}{l}171 \\
0\end{array}$ & $100.0 \%$ & $100.0 \%$ & \\
\hline
\end{tabular}

Of the studied cases, 1270(74.3\%) were smokers. Males included $811(81.9 \%)$ cases and females included $459(63.8 \%)$ cases. Males were more as compared to females with as statistical significance $(\mathrm{p}=0.0001)$. The association of smoking with cardiac conduction defects was found to be significant $(\mathrm{p}=0.0001)$.

Table 11:- Showing the distribution and percentage of smoking in different cardiac conduction defects in studied cases.

\begin{tabular}{|c|c|c|c|c|}
\hline \multicolumn{2}{|l|}{ DIAGNOSIS } & \multicolumn{2}{|c|}{ SMOKING } & \multirow{3}{*}{\begin{tabular}{|l} 
Total \\
34
\end{tabular}} \\
\hline & & YES & NO & \\
\hline \multirow[t]{2}{*}{ 1st DEGREE } & $\mathrm{n}$ & 15 & 19 & \\
\hline & Row \% & $44.1 \%$ & $55.9 \%$ & $100.0 \%$ \\
\hline \multirow[t]{2}{*}{ 2nd DEGREE TYPE 1} & $\mathrm{n}$ & 38 & 27 & 65 \\
\hline & Row \% & $58.5 \%$ & $41.5 \%$ & $100.0 \%$ \\
\hline \multirow[t]{2}{*}{ 2nd DEGREE TYPE 2} & $\mathrm{n}$ & 19 & 7 & 26 \\
\hline & Row \% & $73.1 \%$ & $26.9 \%$ & $100.0 \%$ \\
\hline \multirow[t]{2}{*}{ CHB } & $\mathrm{n}$ & 98 & 6 & 104 \\
\hline & Row \% & $94.2 \%$ & $5.8 \%$ & $100.0 \%$ \\
\hline \multirow[t]{2}{*}{ LAHB } & $\mathrm{n}$ & 303 & 128 & 431 \\
\hline & Row \% & $70.3 \%$ & $29.7 \%$ & $100.0 \%$ \\
\hline \multirow[t]{2}{*}{ LBBB } & $\mathrm{n}$ & 408 & 93 & 501 \\
\hline & Row \% & $81.4 \%$ & $18.6 \%$ & $100.0 \%$ \\
\hline
\end{tabular}




\begin{tabular}{|l|l|l|l|l|}
\hline \multirow{2}{*}{ LPHB } & $\mathrm{n}$ & 6 & 1 & 7 \\
\cline { 2 - 5 } & Row $\%$ & $85.7 \%$ & $14.3 \%$ & $100.0 \%$ \\
\hline \multirow{2}{*}{ RBBB } & $\mathrm{n}$ & 196 & 112 & 308 \\
\cline { 2 - 5 } & Row \% & $63.6 \%$ & $36.4 \%$ & $100.0 \%$ \\
\hline \multirow{2}{*}{ RBBB + LAH } & $\mathrm{n}$ & 128 & 33 & 161 \\
\cline { 2 - 5 } & Row \% & $79.5 \%$ & $20.5 \%$ & $100.0 \%$ \\
\hline \multirow{2}{*}{ RBBB + LPH } & $\mathrm{n}$ & 6 & 6 & 12 \\
\cline { 2 - 5 } & Row \% & $50.0 \%$ & $50.0 \%$ & $100.0 \%$ \\
\hline \multirow{2}{*}{ SI SII SIII } & $\mathrm{n}$ & 10 & 3 & 13 \\
\cline { 2 - 5 } & Row \% & $76.9 \%$ & $23.1 \%$ & $100.0 \%$ \\
\hline \multirow{2}{*}{ TFB } & $\mathrm{n}$ & 43 & 5 & 48 \\
\cline { 2 - 5 } & Row \% & $89.6 \%$ & $10.4 \%$ & $100.0 \%$ \\
\hline \multirow{2}{*}{ Total } & $\mathrm{n}$ & 1270 & 440 & 1710 \\
\cline { 2 - 5 } & Row \% & $74.3 \%$ & $25.7 \%$ & $100.0 \%$ \\
\hline
\end{tabular}

The percentage of smoking in different cardiac conduction defects in decreasing order is as under:
I. Complete heart block (CHB) :
$94.2 \%$
II. Trifascicular heart block(TFB) :
$89.6 \%$
III. Left posterior hemiblock( LPHB) :
$85.7 \%$
IV. Left bundle branch block( LBBB):
$81.4 \%$
V. Bifascicular block ( RBBB +LAH, RBBB + LPH) : $77.4 \%$
VI. $\quad$ SI SII SIII :
$76.9 \%$
$73.1 \%$
VII. $\quad 2^{\text {nd }}$ degree type 2 :
$70.3 \%$
VIII. Left anterior hemiblock ( LAHB) :
$\begin{array}{cll}\text { IX. } & \text { Right bundle branch block( RBBB) : } & 63.6 \% \\ \text { X. } & 2^{\text {nd }} \text { degree type 1: } & 58.5 \%\end{array}$
XI. $\quad 1^{\text {st }}$ degree heart block :
$44.1 \%$

Table 12:- Showing the distribution of the studied cases as per the presence or absence of sedentary life style.

\begin{tabular}{|c|c|c|c|c|c|c|c|c|c|c|}
\hline \multirow{3}{*}{$\begin{array}{l}\text { Sedentar } \\
\text { y } \\
\text { Lifestyle }\end{array}$} & \multicolumn{9}{|c|}{ SEX } & \multirow{2}{*}{$\begin{array}{l}\mathbf{P} \\
\text { valu }\end{array}$} \\
\hline & \multicolumn{3}{|c|}{ MALE } & \multicolumn{3}{|c|}{ FEMALE } & \multicolumn{3}{|c|}{ Total } & \\
\hline & $\mathrm{n}$ & $\begin{array}{l}\text { Row } \\
\%\end{array}$ & $\begin{array}{l}\text { Colum } \\
\text { n \% }\end{array}$ & $\mathrm{n}$ & $\begin{array}{l}\text { Row } \\
\%\end{array}$ & $\begin{array}{l}\text { Colum } \\
\text { n \% }\end{array}$ & $\mathrm{n}$ & $\begin{array}{l}\text { Row } \\
\%\end{array}$ & $\begin{array}{l}\text { Colum } \\
\text { n \% }\end{array}$ & \multirow{4}{*}{$\begin{array}{l}0.00 \\
1\end{array}$} \\
\hline YES & $\begin{array}{l}28 \\
1\end{array}$ & $\begin{array}{l}44.5 \\
\%\end{array}$ & $28.4 \%$ & $\begin{array}{l}35 \\
1\end{array}$ & $\begin{array}{l}55.5 \\
\%\end{array}$ & $48.8 \%$ & 632 & $\begin{array}{l}100.0 \\
\%\end{array}$ & $37.0 \%$ & \\
\hline NO & $\begin{array}{l}70 \\
9\end{array}$ & $\begin{array}{l}65.8 \\
\%\end{array}$ & $71.6 \%$ & $\begin{array}{l}36 \\
9\end{array}$ & $\begin{array}{l}34.2 \\
\%\end{array}$ & $51.2 \%$ & $\begin{array}{l}107 \\
8\end{array}$ & $\begin{array}{l}100.0 \\
\%\end{array}$ & $63.0 \%$ & \\
\hline Total & $\begin{array}{l}99 \\
0\end{array}$ & $\begin{array}{l}57.9 \\
\%\end{array}$ & $100.0 \%$ & $\begin{array}{l}72 \\
0\end{array}$ & $\begin{array}{l}42.1 \\
\%\end{array}$ & $100.0 \%$ & $\begin{array}{l}171 \\
0\end{array}$ & $\begin{array}{l}100.0 \\
\%\end{array}$ & $100.0 \%$ & \\
\hline
\end{tabular}

Of the studied cases, $632(37 \%)$ were living a sedentary life. Males included 281 (28.4\%) cases and females included 351(48.8\%).Females were significantly more as compared to females with a $\mathrm{p}$ value of 0.001 . The association of sedentary life style with cardiac conduction defects was found to be statistically significant. $(\mathrm{p}=0.001)$.

Table 13:- Showing the distribution and percentage of cases living a sedentary life style in different cardiac conduction defects.

\begin{tabular}{|l|l|l|l|l|}
\hline \multirow{2}{*}{ Diagnosis } & \multicolumn{2}{l|}{ Sedentary lifestyle } & \multirow{2}{*}{ Total } \\
\cline { 3 - 5 } \multicolumn{2}{c|}{} & Yes & No & \\
\hline \multirow{2}{*}{ 1st DEGREE } & $\mathrm{n}$ & 0 & 34 & 34 \\
\cline { 2 - 5 } & Row $\%$ & $0.0 \%$ & $100.0 \%$ & $100.0 \%$ \\
\hline \multirow{2}{*}{ 2nd DEGREE TYPE 1 } & $\mathrm{n}$ & 7 & 58 & 65 \\
\cline { 2 - 5 } & Row \% & $10.8 \%$ & $89.2 \%$ & $100.0 \%$ \\
\hline \multirow{2}{*}{ 2nd DEGREE TYPE 2 } & $\mathrm{n}$ & 9 & 17 & 26 \\
\cline { 2 - 5 } & Row \% & $34.6 \%$ & $65.4 \%$ & $100.0 \%$ \\
\hline
\end{tabular}




\begin{tabular}{|c|c|c|c|c|}
\hline \multirow[t]{2}{*}{ CHB } & $\mathrm{n}$ & 52 & 52 & 104 \\
\hline & Row \% & $50.0 \%$ & $50.0 \%$ & $100.0 \%$ \\
\hline \multirow[t]{2}{*}{ LAHB } & $\mathrm{n}$ & 143 & 288 & 431 \\
\hline & Row $\%$ & $33.2 \%$ & $66.8 \%$ & $100.0 \%$ \\
\hline \multirow[t]{2}{*}{ LBBB } & $\mathrm{n}$ & 250 & 251 & 501 \\
\hline & Row \% & $49.9 \%$ & $50.1 \%$ & $100.0 \%$ \\
\hline \multirow[t]{2}{*}{ LPHB } & $\mathrm{n}$ & 3 & 4 & 7 \\
\hline & Row \% & $42.9 \%$ & $57.1 \%$ & $100.0 \%$ \\
\hline \multirow[t]{2}{*}{ RBBB } & $\mathrm{n}$ & 77 & 231 & 308 \\
\hline & Row \% & $25.0 \%$ & $75.0 \%$ & $100.0 \%$ \\
\hline \multirow[t]{2}{*}{ RBBB + LAH } & $\mathrm{n}$ & 53 & 108 & 161 \\
\hline & Row \% & $32.9 \%$ & $67.1 \%$ & $100.0 \%$ \\
\hline \multirow[t]{2}{*}{$\mathrm{RBBB}+\mathrm{LPH}$} & $\mathrm{n}$ & 3 & 9 & 12 \\
\hline & Row \% & $25.0 \%$ & $75.0 \%$ & $100.0 \%$ \\
\hline \multirow[t]{2}{*}{ SI SII SIII } & $\mathrm{n}$ & 3 & 10 & 13 \\
\hline & Row \% & $23.1 \%$ & $76.9 \%$ & $100.0 \%$ \\
\hline \multirow[t]{2}{*}{ TFB } & $\mathrm{n}$ & 32 & 16 & 48 \\
\hline & Row \% & $66.7 \%$ & $33.3 \%$ & $100.0 \%$ \\
\hline \multirow[t]{2}{*}{ Total } & $\mathrm{n}$ & 632 & 1078 & 1710 \\
\hline & Row \% & $37.0 \%$ & $63.0 \%$ & $100.0 \%$ \\
\hline
\end{tabular}

The percentage of sedentary life style in different cardiac conduction defects in decreasing order is as under:
I. Trifascicular heart block(TFB) :
$66.7 \%$
II. Complete heart block (CHB) :
$50.0 \%$
III. Left bundle branch block( LBBB) :
$49.9 \%$
IV. Left posterior hemiblock( LPHB) :
$42.9 \%$
V. $\quad 2^{\text {nd }}$ degree type 2 :
$34.6 \%$
VI. Left anterior hemiblock ( LAHB) :
$33.2 \%$
VII. Bifascicular block ( RBBB +LAH, RBBB + LPH) : $32.3 \%$
VIII. Right bundle branch block( RBBB): $\quad 25.0 \%$
IX. SI SII SIII :
$23.1 \%$
X. $\quad 2^{\text {nd }}$ degree type 1 :
$10.8 \%$
XI. $1^{\text {st }}$ degree heart block :
0

Table 14:- Showing the distribution of the studied cases as per the presence or absence of obesity.

\begin{tabular}{|c|c|c|c|c|c|c|c|c|c|c|}
\hline \multirow[t]{2}{*}{ Obesity } & \multicolumn{3}{|c|}{ Male } & \multicolumn{3}{|c|}{ Female } & \multicolumn{3}{|c|}{ Total } & \\
\hline & $\mathbf{n}$ & $\begin{array}{l}\text { Row } \\
\%\end{array}$ & $\begin{array}{l}\text { Column } \\
\%\end{array}$ & $\mathbf{n}$ & $\begin{array}{l}\text { Row } \\
\%\end{array}$ & $\begin{array}{l}\text { Column } \\
\%\end{array}$ & n & $\begin{array}{l}\text { Row } \\
\%\end{array}$ & $\begin{array}{l}\text { Column } \\
\%\end{array}$ & \multirow{4}{*}{0.006} \\
\hline Yes & 230 & $46.3 \%$ & $23.2 \%$ & 266 & $53.7 \%$ & $36.9 \%$ & 496 & $100.0 \%$ & $29.0 \%$ & \\
\hline No & 760 & $51.5 \%$ & $76.8 \%$ & 454 & $48.5 \%$ & $63.1 \%$ & 1214 & $100.0 \%$ & $71.0 \%$ & \\
\hline Total & 990 & $57.9 \%$ & $100.0 \%$ & 720 & $42.1 \%$ & $100.0 \%$ & 1710 & $100.0 \%$ & $100.0 \%$ & \\
\hline
\end{tabular}

Of the studied cases, 496 (29\%) were obese. Females were more 266 (36.9\%) cases as compared to males $230(23.2 \%)$ and the difference was statistically significant $(\mathrm{p}=0.001)$ The presence of obesity in the studied population was found to be significant $(\mathrm{p}=0.006)$.

Table 15:- Showing the distribution and percentage of obesity in different cardiac conduction defects in studied cases.

\begin{tabular}{|l|l|l|l|l|}
\hline \multicolumn{2}{|c|}{ DIAGNOSIS } & OBESITY & \multirow{2}{*}{ Total } \\
\cline { 3 - 5 } \multicolumn{2}{|c|}{} & YES & NO & \\
\hline \multirow{2}{*}{1 st DEGREE } & $\mathrm{n}$ & 7 & 27 & 34 \\
\cline { 2 - 5 } & Row \% & $20.5 \%$ & $79.5 \%$ & $100.0 \%$ \\
\hline \multirow{2}{*}{ 2nd DEGREE TYPE 1 } & $\mathrm{n}$ & 16 & 49 & 65 \\
\cline { 2 - 5 } & Row \% & $24.6 \%$ & $75.4 \%$ & $100.0 \%$ \\
\hline
\end{tabular}




\begin{tabular}{|l|l|l|l|l|}
\hline \multirow{2}{*}{ 2nd DEGREE TYPE 2 } & $\mathrm{n}$ & 14 & 12 & 26 \\
\cline { 2 - 5 } & Row $\%$ & $53.8 \%$ & $46.2 \%$ & $100.0 \%$ \\
\hline \multirow{2}{*}{ CHB } & $\mathrm{n}$ & 40 & 64 & 104 \\
\cline { 2 - 5 } & Row $\%$ & $38.4 \%$ & $61.6 \%$ & $100.0 \%$ \\
\hline \multirow{2}{*}{ LBBB } & $\mathrm{n}$ & 83 & 348 & 431 \\
\cline { 2 - 5 } & Row \% & $19.2 \%$ & $80.8 \%$ & $100.0 \%$ \\
\hline \multirow{2}{*}{ LPHB } & $\mathrm{n}$ & 171 & 330 & 501 \\
\cline { 2 - 5 } & Row $\%$ & $34.0 \%$ & $66.0 \%$ & $100.0 \%$ \\
\hline \multirow{2}{*}{ RBBB } & $\mathrm{n}$ & 4 & 3 & 7 \\
\cline { 2 - 5 } & Row \% & $57.1 \%$ & $42.9 \%$ & $100.0 \%$ \\
\hline \multirow{2}{*}{ RBBB + LAH } & $\mathrm{n}$ & 75 & 233 & 308 \\
\cline { 2 - 5 } & Row \% & $24.3 \%$ & $75.7 \%$ & $100.0 \%$ \\
\hline \multirow{2}{*}{ RBBB + LPH } & $\mathrm{n}$ & 59 & 102 & 161 \\
\cline { 2 - 5 } & Row \% & $36.6 \%$ & $63.4 \%$ & $100.0 \%$ \\
\hline \multirow{2}{*}{ SI SII SIII } & $\mathrm{n}$ & 2 & 10 & 12 \\
\cline { 2 - 5 } & Row \% & $16.7 \%$ & $83.3 \%$ & $100.0 \%$ \\
\hline \multirow{2}{*}{ TFB } & $\mathrm{n}$ & 5 & 8 & 13 \\
\cline { 2 - 5 } & Row \% & $38.0 \%$ & $62.0 \%$ & $100.0 \%$ \\
\hline Total & $\mathrm{n}$ & 20 & 28 & 48 \\
\cline { 2 - 5 } & Row \% & $41.6 \%$ & $58.4 \%$ & $100.0 \%$ \\
\hline
\end{tabular}

The percentage of obesity in different cardiac conduction defects in decreasing order is as under:
I. Left posterior hemiblock( LPHB):
$57.1 \%$
II. $\quad 2^{\text {nd }}$ degree type 2 :
$53.8 \%$
III. Trifascicular heart block(TFB):
$41.6 \%$
IV. Complete heart block (CHB):
$38.4 \%$
V. SI SII SIII :
$38.0 \%$
VI. Bifascicular block ( RBBB +LAH, RBBB + LPH): $\quad 35.2 \%$
VII. Left bundle branch block( LBBB):
$34.0 \%$
VIII. $\quad 2^{\text {nd }}$ degree type 1:
$24.6 \%$
IX. Right bundle branch block( RBBB):
$24.3 \%$
X. $\quad 1^{\text {st }}$ degree heart block :
$20.5 \%$
XI. Left anterior hemiblock ( LAHB):
$19.2 \%$

Table 16:- Showing the distribution of the studied cases as per the presence or absence of dyslipidemias

\begin{tabular}{|c|c|c|c|c|c|c|c|c|c|c|}
\hline \multirow{3}{*}{$\begin{array}{l}\text { Dyslipide } \\
\text { mia }\end{array}$} & \multicolumn{9}{|l|}{ Sex } & \multirow{3}{*}{$\begin{array}{l}\mathbf{P} \\
\text { valu } \\
\text { e }\end{array}$} \\
\hline & \multicolumn{3}{|c|}{ Male } & \multicolumn{3}{|c|}{ Female } & \multicolumn{3}{|c|}{ Total } & \\
\hline & $\begin{array}{l}\text { Cou } \\
\text { nt }\end{array}$ & $\begin{array}{l}\text { Row } \\
\%\end{array}$ & $\begin{array}{l}\text { Colu } \\
\text { mn } \\
\%\end{array}$ & $\begin{array}{l}\text { Cou } \\
\text { nt }\end{array}$ & $\begin{array}{l}\text { Row } \\
\%\end{array}$ & $\begin{array}{l}\text { Colu } \\
\text { mn } \\
\%\end{array}$ & $\begin{array}{l}\text { Cou } \\
\text { nt }\end{array}$ & $\begin{array}{l}\text { Row } \\
\%\end{array}$ & $\begin{array}{l}\text { Column } \\
\%\end{array}$ & \\
\hline YES & 181 & $\begin{array}{l}42.2 \\
\%\end{array}$ & $18.3 \%$ & 248 & $\begin{array}{l}57.8 \\
\%\end{array}$ & $34.4 \%$ & 429 & $\begin{array}{l}100.0 \\
\%\end{array}$ & $25.1 \%$ & $\begin{array}{l}\mathbf{0 . 0 0} \\
1\end{array}$ \\
\hline NO & 809 & $\begin{array}{l}63.2 \\
\% \\
\end{array}$ & $81.7 \%$ & 472 & $\begin{array}{l}36.8 \\
\%\end{array}$ & $65.6 \%$ & 1281 & $\begin{array}{l}100.0 \\
\%\end{array}$ & $74.9 \%$ & \\
\hline Total & 990 & $\begin{array}{l}57.9 \\
\% \\
\end{array}$ & $\begin{array}{l}100.0 \\
\%\end{array}$ & 720 & $\begin{array}{l}42.1 \\
\%\end{array}$ & $\begin{array}{l}100.0 \\
\%\end{array}$ & 1710 & $\begin{array}{l}100.0 \\
\%\end{array}$ & $100.0 \%$ & \\
\hline
\end{tabular}

Of all the studied cases, 429 (25.1\%) were having dyslipidemia. Males included 181(18.3\%) and females included $248(34.4 \%)$.Females were more as compared to males and the difference was statistically significant $(\mathrm{p}=0.001)$. The association of Dyslipidemia with cardiac conduction defect was found to be significant $(\mathrm{p}=0.001)$. 
Table 17:- Showing the distribution and percentage of Dyslipidemia in different cardiac conduction defects in studied cases.

\begin{tabular}{|c|c|c|c|c|}
\hline \multicolumn{2}{|l|}{ Diagnosis } & \multicolumn{2}{|c|}{ Dyslipidemia } & \multirow{3}{*}{$\begin{array}{l}\text { Total } \\
34\end{array}$} \\
\hline & & \multirow{2}{*}{$\frac{\text { Yes }}{6}$} & \multirow{2}{*}{$\begin{array}{c}\text { No } \\
28\end{array}$} & \\
\hline $1^{\text {st }}$ DEGREE & $\mathrm{n}$ & & & \\
\hline & Row \% & $17.6 \%$ & $82.4 \%$ & $100.0 \%$ \\
\hline \multirow[t]{2}{*}{$2^{\text {nd }}$ DEGREE TYPE 1} & $\mathrm{n}$ & 13 & 52 & 65 \\
\hline & Row \% & $20.0 \%$ & $80.0 \%$ & $100.0 \%$ \\
\hline \multirow[t]{2}{*}{$2^{\text {nd }}$ DEGREE TYPE 2} & $\mathrm{n}$ & 12 & 14 & 26 \\
\hline & Row \% & $46.2 \%$ & $53.8 \%$ & $100.0 \%$ \\
\hline \multirow[t]{2}{*}{$\mathrm{CHB}$} & $\mathrm{n}$ & 28 & 76 & 104 \\
\hline & Row $\%$ & $26.9 \%$ & $73.1 \%$ & $100.0 \%$ \\
\hline \multirow[t]{2}{*}{ LAHB } & $\mathrm{n}$ & 80 & 351 & 431 \\
\hline & Row \% & $18.6 \%$ & $81.4 \%$ & $100.0 \%$ \\
\hline \multirow[t]{2}{*}{ LBBB } & $\mathrm{n}$ & 149 & 352 & 501 \\
\hline & Row $\%$ & $29.7 \%$ & $70.3 \%$ & $100.0 \%$ \\
\hline \multirow[t]{2}{*}{ LPHB } & $\mathrm{n}$ & 4 & 3 & 7 \\
\hline & Row $\%$ & $57.1 \%$ & $42.9 \%$ & $100.0 \%$ \\
\hline \multirow[t]{2}{*}{ RBBB } & $\mathrm{n}$ & 66 & 242 & 308 \\
\hline & Row \% & $21.4 \%$ & $78.6 \%$ & $100.0 \%$ \\
\hline \multirow[t]{2}{*}{ RBBB + LAH } & $\mathrm{n}$ & 46 & 115 & 161 \\
\hline & Row $\%$ & $28.6 \%$ & $71.4 \%$ & $100.0 \%$ \\
\hline \multirow[t]{2}{*}{ RBBB + LPH } & $\mathrm{n}$ & 8 & 4 & 12 \\
\hline & Row \% & $66.7 \%$ & $33.3 \%$ & $100.0 \%$ \\
\hline \multirow[t]{2}{*}{ SI SII SIII } & $\mathrm{n}$ & 4 & 9 & 13 \\
\hline & Row $\%$ & $30.8 \%$ & $69.2 \%$ & $100.0 \%$ \\
\hline \multirow[t]{2}{*}{ TFB } & $\mathrm{n}$ & 13 & 35 & 48 \\
\hline & Row $\%$ & $27.1 \%$ & $72.9 \%$ & $100.0 \%$ \\
\hline \multirow[t]{2}{*}{ Total } & $\mathrm{n}$ & 429 & 1281 & 1710 \\
\hline & Row \% & $25.1 \%$ & $74.9 \%$ & $100.0 \%$ \\
\hline
\end{tabular}

The percentage of Dyslipidemia in different cardiac conduction defects in decreasing order is as under:
I. $\quad$ Left posterior hemiblock( LPHB) :
$57.1 \%$
II. $\quad 2^{\text {nd }}$ degree type 2 :
$46.2 \%$
III. Bifascicular block ( RBBB +LAH, RBBB + LPH) : $31.2 \%$
IV. SI SII SIII :
$30.8 \%$
V. Left bundle branch block( LBBB) :
$29.7 \%$
VI. Trifascicular heart block(TFB) :
$27.1 \%$
VII. Complete heart block (CHB) :
$26.9 \%$
VIII. Right bundle branch block( RBBB) :
$21.4 \%$
IX. $\quad 2^{\text {nd }}$ degree type 1 :
$20.0 \%$
X. Left anterior hemiblock ( LAHB) :
$18.6 \%$
XI. $1^{\text {st }}$ degree heart block :
$17.6 \%$

Table 18:- Showing the distribution of the studied cases as per the presence or absence of hyperuricemia.

\begin{tabular}{|c|c|c|c|c|c|c|c|c|c|c|}
\hline \multirow[t]{3}{*}{ Gout } & \multicolumn{6}{|l|}{ Sex } & & & & \multirow{2}{*}{$\begin{array}{l}\text { P } \\
\text { valu } \\
\text { e }\end{array}$} \\
\hline & \multicolumn{3}{|l|}{ Male } & \multicolumn{3}{|c|}{ Female } & \multicolumn{3}{|l|}{ Total } & \\
\hline & $\begin{array}{l}\text { Coun } \\
t\end{array}$ & $\begin{array}{l}\text { Row } \\
\%\end{array}$ & $\begin{array}{l}\text { Colum } \\
\text { n \% }\end{array}$ & $\begin{array}{l}\text { Coun } \\
t\end{array}$ & $\begin{array}{l}\text { Row } \\
\%\end{array}$ & $\begin{array}{l}\text { Colum } \\
\text { n } \%\end{array}$ & $\begin{array}{l}\text { Coun } \\
t\end{array}$ & Row \% & $\begin{array}{l}\text { Colu } \\
\text { mn } \\
\%\end{array}$ & 0.85 \\
\hline YES & 30 & $\begin{array}{l}58.8 \\
\%\end{array}$ & $3.0 \%$ & 21 & $41.2 \%$ & $2.9 \%$ & 51 & $100.0 \%$ & $3.0 \%$ & \\
\hline NO & 960 & $\begin{array}{l}57.9 \\
\%\end{array}$ & $97.0 \%$ & 699 & $42.1 \%$ & $97.1 \%$ & 1659 & $100.0 \%$ & $\begin{array}{l}97.0 \\
\%\end{array}$ & \\
\hline
\end{tabular}




\begin{tabular}{|l|l|l|l|l|l|l|l|l|l|l|}
\hline $\begin{array}{l}\text { Tota } \\
1\end{array}$ & 990 & $\begin{array}{l}57.9 \\
\%\end{array}$ & $100.0 \%$ & 720 & $42.1 \%$ & $\begin{array}{l}100.0 \\
\%\end{array}$ & 1710 & $100.0 \%$ & $\begin{array}{l}100.0 \\
\%\end{array}$ \\
\hline
\end{tabular}

Of the studied cases, 51 (3\%) were hyperuricemics. Males included 30 (3\%) cases and females included 21(2.9\%).Males were more as compared to females with no statistically significant difference $(\mathrm{p}=0.89)$. The presence of hyperuricemia in the studied population was found to be non significant $(\mathrm{p}=0.85)$.

Table 19:- Showing the distribution and percentage of hyperuricemia in different cardiac conduction defects in studied cases.

\begin{tabular}{|c|c|c|c|c|}
\hline \multirow{2}{*}{\multicolumn{2}{|c|}{ DIAGNOSIS }} & \multicolumn{2}{|c|}{ HYPERURICEMIA } & \multirow{3}{*}{$\begin{array}{l}\text { Total } \\
34 \\
\end{array}$} \\
\hline & & \multirow{2}{*}{$\begin{array}{l}\text { YES } \\
0\end{array}$} & \multirow{2}{*}{ NO } & \\
\hline \multirow[t]{2}{*}{ 1st DEGREE } & $\mathrm{n}$ & & & \\
\hline & Row \% & $0.0 \%$ & $100.0 \%$ & $100.0 \%$ \\
\hline \multirow[t]{2}{*}{ 2nd DEGREE TYPE 1} & $\mathrm{n}$ & 1 & 64 & 65 \\
\hline & Row \% & $1.5 \%$ & $98.5 \%$ & $100.0 \%$ \\
\hline \multirow[t]{2}{*}{ 2nd DEGREE TYPE 2} & $\mathrm{n}$ & 3 & 23 & 26 \\
\hline & Row \% & $11.5 \%$ & $88.5 \%$ & $100.0 \%$ \\
\hline \multirow[t]{2}{*}{ CHB } & $\mathrm{n}$ & 3 & 101 & 104 \\
\hline & Row \% & $2.9 \%$ & $97.1 \%$ & $100.0 \%$ \\
\hline \multirow[t]{2}{*}{ LAHB } & $\mathrm{n}$ & 14 & 417 & 431 \\
\hline & Row \% & $3.2 \%$ & $96.8 \%$ & $100.0 \%$ \\
\hline \multirow[t]{2}{*}{ LBBB } & $\mathrm{n}$ & 17 & 484 & 501 \\
\hline & Row \% & $3.4 \%$ & $96.6 \%$ & $100.0 \%$ \\
\hline \multirow[t]{2}{*}{ LPHB } & $\mathrm{n}$ & 0 & 7 & 7 \\
\hline & Row \% & $0.0 \%$ & $100.0 \%$ & $100.0 \%$ \\
\hline \multirow[t]{2}{*}{ RBBB } & $\mathrm{n}$ & 6 & 302 & 308 \\
\hline & Row \% & $1.9 \%$ & $98.1 \%$ & $100.0 \%$ \\
\hline \multirow[t]{2}{*}{ RBBB + LAH } & $\mathrm{n}$ & 2 & 159 & 161 \\
\hline & Row \% & $1.2 \%$ & $98.8 \%$ & $100.0 \%$ \\
\hline \multirow[t]{2}{*}{ RBBB + LPH } & $\mathrm{n}$ & 1 & 11 & 12 \\
\hline & Row \% & $8.3 \%$ & $91.7 \%$ & $100.0 \%$ \\
\hline \multirow[t]{2}{*}{ SI SII SIII } & $\mathrm{n}$ & 0 & 13 & 13 \\
\hline & Row \% & $0.0 \%$ & $100.0 \%$ & $100.0 \%$ \\
\hline \multirow[t]{2}{*}{ TFB } & $\mathrm{n}$ & 4 & 44 & 48 \\
\hline & Row $\%$ & $8.3 \%$ & $91.7 \%$ & $100.0 \%$ \\
\hline \multirow[t]{2}{*}{ Total } & $\mathrm{n}$ & 51 & 1659 & 1710 \\
\hline & Row \% & $3.0 \%$ & $97.0 \%$ & $100.0 \%$ \\
\hline
\end{tabular}

The percentage of hyperuricemia in different cardiac conduction defects in decreasing order is as under:

I. $\quad 2^{\text {nd }}$ degree type $2:$

II. Trifascicular heart block(TFB) : $\quad 8.3 \%$

III. Left bundle branch block( LBBB) : $\quad 3.4 \%$

IV. Left anterior hemiblock ( LAHB) : $\quad 3.2 \%$

V. Complete heart block (CHB): $\quad 2.9 \%$

VI. Right bundle branch block( RBBB) : $\quad 1.9 \%$

VII. Bifascicular block ( RBBB +LAH, RBBB + LPH) : $1.73 \%$

VIII. $\quad 2^{\text {nd }}$ degree type 1 :

IX. Left posterior hemiblock( LPHB) : $\quad 0$

X. SI SII SIII :

XI. $\quad 1^{\text {st }}$ degree heart block :

0

\section{Discussion:-}

Cardiac conduction abnormalities vary with population, age (being lowest in young and highest in elderly), from symptomatic to asymptomatic and from male to female. A number of cardiovascular risk factors form a risk of cardiac conduction abnormalities also. 
We conducted this hospital based study on 1710 patients to find out the relationship of cardiac conduction defects with underlying cardiovascular risk factors.

\section{Risk Factors:- \\ Hypertension:-}

In our study hypertension was present in $64 \%$ of cases and the association of hypertension with different cardiac conduction defects was found to be highly statistically significant $(\mathrm{p}<0.0001)$. Our observation that hypertension is one of the commonest cause of conduction defects is in conformity with the observations made by Yardena $\mathrm{S}^{12}$, Wani BA ${ }^{13}$, Barbara V. Howard ${ }^{14,}$ Lone $\mathrm{NA}^{15}$, Najar MS ${ }^{16}$. Najar MS ${ }^{16}$ reported hypertension in $48 \%$ of cases and Risteard Mulchay et $\mathrm{al}^{17}$ reported hypertension in $59 \%$ of cases. In contrast, Leon d. Ostrander $\mathrm{JR}^{18}$ reported hypertension in $31 \%$ of cases.

\section{Diabetes Mellitus:-}

Diabetes mellitus (DM) is a major risk factor for cardiovascular disease and mortality with increasing prevalence in the ageing population. In our study we found the prevalence of diabetes in the studied population as $24 \%$. The association of diabetes with the cardiac conduction abnormalities was found to be significant ( $\mathrm{p}=0.030$ ). Arvo JO et $\mathrm{al}^{19}$ in The strong Heart Study reported diabetes in $28 \%$ of cases. Perlman et $\mathrm{al}^{6}$ reported diabetes in $22 \%$ and Ostrander et $\mathrm{al}^{18}$ reported diabetes in $17 \%$ of cases. However in contrast Risteard Mulchay et $\mathrm{al}^{17}$ and Najar $\mathrm{MS}^{16}$ reported diabetes in $11 \%$ and $6 \%$ respectively.

\section{Coronary Artery Disease (Cad);-}

In our study we found the prevalence of coronary artery disease in our population as $26 \%$ and it was significantly associated with cardiac conduction defects with a $\mathrm{p}$ value of 0.074 . John $\mathrm{H}$ McAnulty et $\mathrm{al}^{20}$ in 1978 on a study of 277 patients reported CAD in $56 \%$ of cases. Risteard Mulchay et $\mathrm{al}^{17}$ reported CAD in $49 \%$ cases. Robert $\mathrm{WL}^{21}$ et al reported presence of CAD in $35 \%$ of cases of conduction defects. Najar $\mathrm{MS}^{16}$ reported presence of CAD in $30 \%$ cases. In contrast, Wani $\mathrm{BA}^{13}$ reported $\mathrm{CAD}$ in $12 \%$ of cases.

\section{Smoking:-}

In our study we found $74.3 \%$ of cases as smokers and the association of smoking was found to be significant $(\mathrm{p}=0.0001)$. Najar $\mathrm{MS}^{16}$ reported smoking in $76 \%$, Lone $\mathrm{NA}^{15}$ in $58 \%$, Ostrander et $\mathrm{al}^{18}$ in $45 \%$. Wani BA et al ${ }^{13}$ found no association between cardiac conduction defect and smoking.

\section{Sedentary Life Style:-}

In our study $36.9 \%$ of the cases were living a sedentary life style and the association of sedentary life style with cardiac conduction defects was found to be significant. $(\mathrm{p}=0.001)$. Najar $\mathrm{MS}^{16}$ found $60 \%$ of the cases in a study of 50 hospitalised patients to be living a sedentary life style. Warren TY et $\mathrm{al}^{22}$ also found a significant relationship between cardiac conduction defects and sedentary life style.

\section{Obesity:-}

In our study, we found $29 \%$ cases to be obese and the association of obesity with the conduction abnormality was found to be significant $(\mathrm{p}=0.001)$. Lone $\mathrm{NA}^{15}$ reported obesity in $47 \%$ of cases, Najar MS ${ }^{16}$ in $34 \%$ and Perlman et $\mathrm{al}^{6}$ reported obesity in $26 \%$ cases. In contrast, Ostrander et $\mathrm{al}^{18}$ reported obesity in only $6 \%$ cases.

\section{Dyslipidemia:-}

In our study, we found the prevalence of dyslipidemics as $25.1 \%$ and the association of dyslipidemia was found to be significant. $(\mathrm{p}=0.001)$. Ostrander et $\mathrm{al}^{18}$ reported dyslipidemia in $25 \%$ of cases. Perlman et $\mathrm{al}^{6}$ in $21 \%$ cases. In contrast, Najar $\mathrm{MS}^{16}$ found dyslipidemia in $14 \%$ of cases.

\section{Hyperuricemia:-}

In our study ,3\% Of cases were hyperuricemic . The association of hyperuricemia with cardiac conduction defect was found to be nonsignificant $\left(\mathrm{p}=0.89\right.$ ). Najar $\mathrm{MS}^{16}$ found hyperuricemia in $2 \%$ of cases .Yamaguchi et al found hyperuricemia in 0.175 of cases. 


\section{Bibliography:-}

1. Charles Fish-Electrocardiography and Vectorcardiography in Braunwald Heart Disease, A textbook of cardiovascular medicine, 4th Edition Vol 1.

2. Hurst's The Heart; 11th Edition p 893-895.

3. Kellkar PN, Elizabeth J. Intraventricular conduction disturbances. JAPI I996; 44(6):402-406.

4. Pryor R and Blount SG. The c linical significance of true left axis deviation, left intraventricular block. Am. Heart Journal 1966; 70:391.

5. Harrisons Principles of Internal Medicine. $18^{\text {th }}$ Ed.

6. Lawrence V. Perlman et al. an epidemiologic study of first degree atrioventricular Block in Tecumseh, Michigan. Chest 1971;59: 40-46.

7. Assantachai $P$ et al. An electrocardiographic survey of elderly Thai people in the rural community. J Med Assoc Thai. 2002; Dec 85(12) : 1273-1279.

8. Upshaw CB Jr. Comparison of the prevalence of first degree atrioventricular block in African-American and in Caucasian patients: an electrocardiographic study III, J Natl Med Assoc. 2004. Jun ; 96(6): 756-760.

9. Guidelines for Data Processing and Analysis of the International Physical Activity Questionnaire(IPAQ). November 2005. www.ipaq.ki.se.

10. Siedell JC. Obesity, insulin Resistance and diabetes- world epidemic. Br. J 2000; 183:5-8.

11. http:/www.igovernment.in/site/India-reworks-obesity-guidelines-BMI lowered / Nov 2008.

12. Yardena S, Irigra et al. Intraventricualar conduction disturbances: A review of prevalence, etiology and progression for 10 years within a stable population of Israeli adult males. American Heart Journal 1978;96(5):669-79.

13. Wani BA, Zargar AH. Pattern of Heart Block in Kashmir- An electrocardiographic Analysis in 3600 Hospitalized patients. Paper presented during llnd National conference on Cardiac Pacing, Calcutta March 3-5, 1983.

14. Charles F MD. Electrocardiograms in the aged: An independent Marker of heart disease, the Am J Of Med, Jan 1981;70:4-5.

15. Lone NA. a study of intraventricular cardiac conduction defects in an adult asymptomatic Kashmiri Population, thesis presented to university of Kashmir 1998;1-70.

16. Najar MS . Clinical profile of intraventricular conduction defects in hospitalized patients in Kashmir, Thesis submitted of university of Kashmir. 1986:1-72.

17. Risteard M, Noel H, Brain M. Aetiology of Bundle Branch Block, Brit Heart J 1968;30:34.

18. Ostrander LD, Ralph LB, Marcus OK, Frederick HE. Electrocardiographic Findings Among the Adult population of a Total Natural Community, Tecumseh, Michigan. Circulation 1965;31:888-97.

19. Arvo JO, Mark D, Richard BD et al. Major Electrocardiographic Abnormalities Among American Indians Aged 45 to 74 years (The strong Heart Study) Am J Cardiol 1996;78:1400-1405.

20. John Mc Anulty, Kmifl'man RN, Edward M, Donald OK, Shahabudin HR. Survival in patients with Intraventricular conduction Defects. Arch Intern Med Jan 1973; 138:30-35.

21. Robert WL, Reed JC, David CV et al. Bundle Branch Block. A review of 100 cases. Am heart J 1947;33:730.

22. Warren TY et al. sedentary behaviors increase risk of cardiovascular disease mortality in men. Med Sci Sports Exerc. 2010 May ; 42(5):879-885. 